\title{
Nonlinear PDE Control of Two-Link Flexible Arm with Nonuniform Cross Section
}

\author{
Mustafa Dog̃an ${ }^{1,2}$, Ömer Morgül $^{3}$ \\ Department of Electrical and Electronics Engineering \\ ${ }^{1}$ Bașkent University, ${ }^{2}$ Bogaziçi University, ${ }^{3}$ Bilkent University, Turkey
}

\begin{abstract}
A two-link flexible arm with nonuniform or variable cross-section by design will be considered based on an exact PDE model with boundary conditions. In this research, the nonlinear controller is used to achieve setpoint regulation of the rigid modes as well as suppression of elastic vibrations. The control laws are obtained by energy based Lyapunov approach.
\end{abstract}

\section{INTRODUCTION}

Two main advantages of flexible robot arms are less weight and low energy consumption. However, the structural modelling and the control design of the flexible arms are much more complicated due to nonlinear coupling between elastic and rigid modes during the complex maneuvers especially with high angular velocities. Various methods have been proposed for control of flexible-link manipulators in the literature. Hybrid control of a single flexible-link manipulator using feedback linearization and singular perturbation approach has been used in [1]. Adaptive feedback linearization has been applied successfully for a nonlinear discrete-time model of a single-link flexible manipulator [2]. Singular perturbation theory has also been used for position and force control in [3]. Strain feedback and active vibration control [4], [5] are other approaches different from the integrated structure-control for nonuniform flexible links in [6]. In order to improve the important features of flexible links such that low mass and moments of inertia and high natural frequencies [7], optimal shape design can be investigated. Furthermore, a high fundamental frequency is desired since it implies a large bandwidth that will allow for fast motion without causing serious vibration problems and stable endpoint control [8]. In this research, inspired by the last three approaches in [6], [7], [8], the control of a two-link flexible arm with variable cross-section by design is improved by employing the Lyapunov method. Different from the energy based multi-link flexible robot control proposed in $[9$, ch.2], LaSalle's invariance principle extended for infinite dimension [10] will be used in order to prove the asymptotic stability of the closed loop system without any modal truncation such that the higher order modes will not

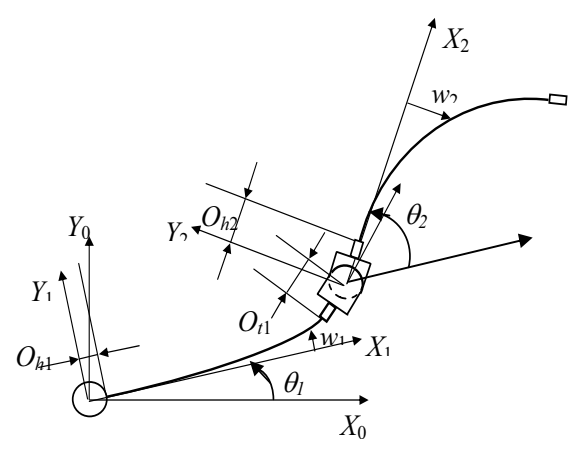

Fig. 1. Arm configuration [4]

be ignored.

Referring to Figure 1 the various symbols represent the following; $X_{o} Y_{o}$ : global inertial system of coordinates; $X_{1} Y_{1}$ : body-fixed system of coordinates attached to undeformed link 1, $X_{2} Y_{2}$ : body-fixed system of coordinates attached to undeformed link 2, $\theta_{1}, \theta_{2}$ : angular displacements of links 1 and 2, $w_{1}, w_{2}$ : flexural displacements of links 1 and 2, $O_{h i}$ : offset of the beam root to the center of the $i^{t h}$ input torque motor, $O_{t i}$ : offset of the beam end to the center of tip mass [4].

\section{Analytical Model}

In this section, an exact model using partial differential equations with boundary conditions is derived using the Hamilton's Principle based on Zhang's work for uniform links [13], [4]. Since the ratio between the length of the beam and its thickness is sufficiently large as proposed in [14], [15], links can be modelled as Euler-Bernoulli beams, which can only be deformed in the flexural direction. The links are modelled in clamped-free configuration, since natural modes of the separated clamped-free links agree very well with actual ones compared to pinned-free configuration [16]. Assuming the manipulator moves in the horizontal plane, in the absence of gravity the potential energy depends only on the flexural deflections.

In order to derive the PDE model, kinetic and potential energy expressions for the links and hubs are obtained. 


\begin{tabular}{|l|l|}
\hline Parameter & Description \\
\hline$E$ & Young's Modulus \\
\hline$I_{i}\left(x_{i}\right)$ & $\begin{array}{l}\text { Variable beam cross section moment } \\
\text { to z axis at the location } x_{i}\end{array}$ \\
\hline$I_{h i}$ & Inertia of $i^{t h}$ hub \\
\hline$I_{t i}$ & Tip inertia of $i^{t h}$ beam \\
\hline$l_{i}$ & Length of $i^{t h}$ link \\
\hline$m_{h i}$ & Mass of $i^{t h}$ hub \\
\hline$m_{t i}$ & Tip mass of $i^{t h}$ beam \\
\hline$x_{i}$ & $\begin{array}{l}\text { Coordinate along the axial center } \\
\text { of the } i^{t h} \text { beam }\end{array}$ \\
\hline$w_{i}\left(x_{i}, t\right)$ & $\begin{array}{l}\text { Transverse movement of point } i \\
\text { at the location } x_{i} \text { of } i^{t h} \text { beam }\end{array}$ \\
\hline$\dot{w}_{i}\left(x_{i}, t\right)$ & $\begin{array}{l}\text { Time rate of transverse movement } \\
\text { of point } i \text { at the location } x_{i} \text { of } i^{t h} \text { beam }\end{array}$ \\
\hline$w_{i x}\left(x_{i}, t\right)$ & $\begin{array}{l}\text { Axial rate of transverse movement } \\
\text { of point } i \text { at the location } x_{i} \text { of } i^{\text {th }} \text { beam }\end{array}$ \\
\hline$\rho_{i}\left(x_{i}\right)$ & $\begin{array}{l}\text { Variable density of the } i^{t h} \text { link } \\
\text { depends on the cross-sectional area at } x_{i}\end{array}$ \\
\hline$\tau_{i}$ & Input torque at $i^{t h}$ motor \\
\hline$\theta_{1}$ & Angular position of the first motor \\
\hline$\hat{\theta}_{2}$ & Angular position of the second motor \\
\hline$\theta_{2}$ & $\theta_{2}=\hat{\theta}_{2}+w_{1 x}\left(l_{1}, t\right)$ \\
\hline
\end{tabular}

TABLE I

PARAMETERS For PDE MOdel [4].

Then, Extended Hamilton's Principle [14] is applied such that

$$
\int_{t_{1}}^{t_{2}}\left(\delta T-\delta V_{s}+\delta W_{n c}\right) d t=0
$$

where $\delta T$ and $\delta V_{s}$ are the variation of total kinetic and potential energy respectively, $\delta W_{n c}$ is the variation of nonconservative work. Thus, the governing equations for a two-link flexible arm with nonuniform cross-section are derived by the variational method and integration by parts and listed below with the notation given in TABLE I:

$$
\begin{array}{r}
\ddot{w}_{1}+\frac{\left(E I_{1}\left(x_{1}\right) w_{1 x x}\right)_{x x}}{\rho_{1}\left(x_{1}\right)}=-x_{1} \ddot{\theta}_{1} \\
\ddot{w}_{2}+\frac{\left(E I_{2}\left(x_{2}\right) w_{2 x x}\right)_{x x}}{\rho_{2}\left(x_{2}\right)}+\operatorname{Cos}_{2}\left[\ddot{w}_{1}\left(l_{1}, t\right)+l_{1} \ddot{\theta}_{1}\right] \\
-\dot{\theta}_{2} \operatorname{Sin} \theta_{2}\left[\dot{w}_{1}\left(l_{1}, t\right)+l_{1} \dot{\theta}_{1}\right]=-x_{2} \ddot{\theta}_{2} \\
I_{h 1} \ddot{\theta}_{1}-E I_{1}(0) w_{1 x x}(0, t)=\tau_{1} \\
I_{t 1} I_{h 2} \ddot{\theta}_{2}-I_{h 2} E I_{1}\left(l_{1}\right) w_{1 x x}\left(l_{1}, t\right) \\
-I_{t 1} E I_{2}\left(l_{2}\right) w_{2 x x}\left(l_{2}, t\right)=\left(I_{t 1}+I_{h 2}\right) \tau_{2} \\
-\int_{0}^{l_{2}} \rho_{2}\left(x_{2}\right) x_{2} d x_{2} \dot{\theta}_{2} \operatorname{Sin}_{2}\left[\dot{w}_{1}\left(l_{1}, t\right)+l_{1} \dot{\theta}_{1}\right] \\
+\int_{0}^{l_{2}} x_{2}\left(E I_{2}\left(x_{2}\right) w_{2 x x}\right)_{x x} d x_{2}
\end{array}
$$

$$
\begin{array}{r}
-I_{h 2}\left[\ddot{\theta}_{1}+\ddot{\theta}_{2}\right]-I_{t 2}\left[\ddot{\theta}_{1}+\ddot{\theta}_{2}+\ddot{w}_{2 x}\left(l_{2}, t\right)\right] \\
-\operatorname{Sin} \theta_{2}\left[\dot{w}_{1}\left(l_{1}, t\right)+l_{1} \dot{\theta}_{1}\right] \int_{0}^{l_{2}} \rho_{2}\left(x_{2}\right) \dot{w}_{2} d x_{2} \\
-m_{t 2} l_{2}\left[l_{2} \ddot{\theta}_{2}+\ddot{w}_{2}\left(l_{2}, t\right)+\operatorname{Cos} \theta_{2}\left[\ddot{w}_{1}\left(l_{1}, t\right)+l_{1} \ddot{\theta}_{1}\right]\right] \\
-m_{t 2} \operatorname{Sin} \theta_{2}\left[\dot{w}_{1}\left(l_{1}, t\right)+l_{1} \dot{\theta}_{1}\right] \dot{w}_{2}\left(l_{2}, t\right) \\
-I_{t 1}\left[\ddot{\theta}_{1}+\ddot{w}_{1 x}\left(l_{1}, t\right)\right]-E I_{1}\left(l_{1}\right) w_{1 x x}\left(l_{1}, t\right)=0 \\
-\int_{0}^{l_{2}} \rho_{2}\left(x_{2}\right)\left\{\left[\ddot{w}_{1}\left(l_{1}, t\right)+l_{1} \ddot{\theta}_{1}\right]\right. \\
\left.+\operatorname{Cos}_{2}\left[\ddot{w}_{2}+x_{2} \ddot{\theta}_{2}\right]-\dot{\theta}_{2} \operatorname{Sin}_{2}\left[\dot{w}_{2}+x_{2} \dot{\theta}_{2}\right]\right\} d x_{2} \\
-\left(m_{t 1}+m_{h 2}+m_{t 2}\right)\left[\ddot{w}_{1}\left(l_{1}, t\right)+l_{1} \ddot{\theta}_{1}\right] \\
+\left[\left(E I_{1}\left(x_{1}\right) w_{1 x x}\right)_{x}\right]_{x_{1}=l_{1}} \\
-m_{t 2}\left\{\operatorname{Cos}_{2}\left[\ddot{w}_{2}\left(l_{2}, t\right)+l_{2} \ddot{\theta}_{2}\right]\right. \\
\left.-\dot{\theta}_{2} \operatorname{Sin}_{2}\left[\dot{w}_{2}\left(l_{2}, t\right)+l_{2} \dot{\theta}_{2}\right]\right\}=0
\end{array}
$$

where all offset values $\left(O_{h i}, O_{t i}\right)$ can be omitted by design and it is assumed that $\theta_{2} \approx \hat{\theta}_{2}$ since the slope at the end of the first link is relatively small. The equations (2) and (3) are the main PDEs(balance of forces) and the equations (4) and (5) are ODEs (conservation of momentum) for rigid coordinates. Equations ( 6 - 10) are the boundary conditions where equation (10) includes the four boundary conditions at the clamped end of the links [17].

We first define the following variables for $i=1,2$

$$
\begin{aligned}
\tau_{b i} & =-E I_{i}(0) w_{i x x}(0, t) \\
\tau_{e i} & =-E I_{i}\left(l_{i}\right) w_{i x x}\left(l_{i}, t\right)
\end{aligned}
$$

where $\tau_{b 1}, \tau_{b 2}$ represent the base strain measured torques and $\tau_{e 1}, \tau_{e 2}$ represent the end strain measured torques for the first and second beams respectively. Boundary conditions ( 6), ( 8) and (9) can be simplified further 
to obtain the following one

$$
\begin{gathered}
\operatorname{Sin} \theta_{2}\left[\dot{w}_{1}\left(l_{1}, t\right)+l_{1} \dot{\theta}_{1}\right]\left\{-\dot{\theta}_{2} \int_{0}^{l_{2}} \rho_{2}\left(x_{2}\right) x_{2} d x_{2}\right. \\
\left.-l_{2} \dot{\theta}_{2} m_{t 2}-\int_{0}^{l_{2}} \rho_{2}\left(x_{2}\right) \dot{w}_{2} d x_{2}-m_{t 2} \dot{w}_{2}\left(l_{2}, t\right)\right\} \\
-\tau_{b 2}+\tau_{e 1}-I_{h 2}\left[\ddot{\theta}_{1}+\ddot{\theta}_{2}\right]-I_{t 1}\left[\ddot{\theta}_{1}+\ddot{w}_{1 x}\left(l_{1}, t\right)\right]=0
\end{gathered}
$$

where the new set of boundary conditions will be (13), ( 7), ( 8) and ( 10).

\section{Controller Design}

The total energy of the system ( 2 - 10) is calculated to get the total energy rate of the system. Then with this insight, the control laws are obtained by Lyapunov approach as given below

$$
\begin{array}{r}
\tau_{11}=\tau_{b 1}+I_{h 1}\left(\frac{-\ddot{w}_{1}\left(l_{1}, t\right)}{l_{1}}-K_{1}\left(\alpha \dot{\theta}_{1}+\beta\left(\theta_{1}-\theta_{1 d}\right)\right)\right) \\
\tau_{21}=\left(I_{h 2} \tau_{e 1}+I_{t 1} \tau_{e 2}+I_{t 1} I_{h 2}\left(\frac{-\ddot{w}_{2}\left(l_{2}, t\right)}{l_{2}}\right.\right. \\
\left.\left.-K_{2}\left(\alpha \dot{\theta}_{2}+\beta\left(\theta_{2}-\theta_{2 d}\right)\right)\right)\right) /\left(I_{t 1}+I_{h 2}\right)
\end{array}
$$

where $K_{1}, K_{2}, \alpha, \beta$ are positive constants and $\theta_{1 d}, \theta_{2 d}$ are the desired positions. Besides, $\ddot{w}_{i}\left(l_{i}, t\right)$ signals in the control laws can be obtained after filtering the output of the wireless accelerometers which are known to be vibration measurement tools. On the other hand, the control laws ( 14 - 15) should be augmented with a parallel controller to ensure asymptotic stability of the closed loop system. In addition to $(14-15)$, we also propose the following control laws

$$
\begin{aligned}
\tau_{12} & =\tau_{b 2}-\tau_{e 2}-K \dot{\theta}_{1} \\
\tau_{22} & =\left(I_{t 1} I_{h 2}\left(\ddot{\theta}_{1}+\ddot{\theta}_{2}\right)+I_{t 1} \tau_{e 2}\right) /\left(I_{t 1}+I_{h 2}\right) \\
\tau_{1} & =\tau_{11}+\gamma_{1} \tau_{12} \\
\tau_{2} & =\tau_{21}+\gamma_{2} \tau_{22}
\end{aligned}
$$

where $K, \gamma_{1}, \gamma_{2}>0$ are proportional gains that are adjusted to make the control laws $\tau_{11}, \tau_{21}$ dominant. This is achieved by choosing small $\gamma_{1}$ and $\gamma_{2}$. The main controller ( 18 - 19) consists of the parallel connection of two separate controllers. Since the system ( 2 - 10) became decoupled and linear by the dominant part of the controller, then additivity and homogeneity properties of linear systems can be applied for the closed-loop system. Thus, the effects of the control laws $\tau_{12}, \tau_{22}$ should be considered independently from the others. Consequently, the main theorem can be introduced as follows

Theorem 3.1: The control laws ( 18 - 19) achieve setpoint regulation of the rigid modes and asymptotic stability of the two-link flexible arm with variable cross-section.

Proof:

The total energy $V$ of the system is a good candidate for Lyapunov function and can be expressed in terms of kinetic and potential energy terms with additional correction term such that

$$
V=T_{1}+T_{2}+T_{3}+T_{4}+T_{5}+T_{6}+V_{s}+\ddot{\theta}_{1}^{2} \geq 0
$$

where $T_{i} \mathrm{~s}$ are the kinetic energy terms of beams, tips and hubs for link 1 and 2 [13]. The total strain potential energy for both links is represented as $V_{s}$. After applying the control laws ( 18 - 19) to the system ( 2 - 10) and after some lengthy but straightforward calculations, the total energy rate of the system is obtained for $M>0$ as follows

$$
\begin{aligned}
\dot{V}<-2\left(K_{1} \beta\right. & {\left.\left[K_{1}(\alpha+\beta M)+\frac{K \gamma_{1}}{I_{h 1}}\right]\right) \dot{\theta}_{1}^{2} } \\
- & 2\left(K_{1} \alpha+\frac{K \gamma_{1}}{I_{h 1}}\right) \ddot{\theta}_{1}^{2}<0
\end{aligned}
$$

Note that the equation ( 21 ) only assures the stability of the closed loop system but does not prove the asymptotic stability. The latter can be shown by using LaSalle's invariance principle extended to infinite dimensional spaces [10]. In order to initiate this part of the proof, equations (2)- (5) should be rewritten in the new coordinates such that

$$
\begin{gathered}
\mathbf{z}=\left[\begin{array}{lllllllll}
z_{1} & z_{2} & z_{3} & z_{4} & z_{5} & z_{6} & z_{7} & z_{8}
\end{array}\right]^{T} \\
=\left[\begin{array}{llllllll}
w_{1} & \dot{w}_{1} & w_{2} & \dot{w}_{2} & \theta_{1}-\theta_{1 d} & \dot{\theta}_{1} & \theta_{2}-\theta_{2 d} & \dot{\theta}_{2}
\end{array}\right]^{T}
\end{gathered}
$$

The system ( 2)- ( 5) can be decomposed to linear and nonlinear parts separately, see [11], such as

$$
\dot{\mathbf{z}}=A \mathbf{z}+f(\mathbf{z})
$$

where $A$ represents the infinite dimensional linear operator and $f$ represents the nonlinear operator. For the linear part of the system (2)- ( 3 ) which is dissipative by feedback controls ( 18 - 19), will be considered in the energy Hilbert space $\mathbf{H}_{i}=H_{E}^{2}\left(0, l_{i}\right) \times L^{2}\left(0, l_{i}\right), H_{E}^{2}\left(0, l_{i}\right)=$ $\left\{w_{i} \in H^{2}\left(0, l_{i}\right) \mid w_{i}(0)=w_{i x}(0)=0\right\}$, in which the inner product induced norm is defined by [12]

$\left\|\left(w_{i}, g_{i}\right)\right\|_{\mathbf{H}_{i}}^{2}=\int_{0}^{l_{i}}\left[\rho_{i}(x)\left|g_{i}(x)\right|^{2}+E I_{i}(x)\left|w_{i x x}(x)\right|^{2}\right] d x$

for $i=1,2$ and $\forall\left(w_{i}, g_{i}\right) \in \mathbf{H}_{i}, 0<x<l_{i}$. Define operator $A_{i}: D\left(A_{i}\right)\left(\subset \mathbf{H}_{i}\right) \rightarrow \mathbf{H}_{i}$ as

$$
\begin{gathered}
A_{i}\left(w_{i}, g_{i}\right)=\left(g_{i}, \frac{-1}{\rho_{i}(x)}\left(E I_{i}(x) w_{i x x}(x)\right)_{x x}\right) \\
D\left(A_{i}\right)=\left\{\left(w_{i}, g_{i}\right) \in\left(H_{E}^{2} \cap H^{4}\right) \times H_{E}^{2}\right\}
\end{gathered}
$$

where $A_{i}^{-1}$ is compact on $\mathbf{H}_{i}$ [12, Lemma 2.1]. We also define the function space

$$
\hat{\mathbf{H}}=\mathbf{H}_{1} \times \mathbf{H}_{2} \times \mathbf{R} \times \mathbf{R} \times \mathbf{R} \times \mathbf{R}
$$


Using the set of equations ( 2 - 10) and integration by parts, we have the linear operator $A: \hat{\mathbf{H}} \rightarrow \hat{\mathbf{H}}$ and the nonlinear operator $f: \hat{\mathbf{H}} \rightarrow \hat{\mathbf{H}}$ are given in equation (22) such as:

$$
\begin{aligned}
& A=\left[\begin{array}{ccc}
A_{1} & 0_{2 \times 2} & 0_{1 \times 4} \\
0_{2 \times 2} & A_{2} & B_{1} \\
0_{4 \times 2} & 0_{4 \times 2} & B_{2}
\end{array}\right] \\
& f(\mathbf{z})=\left[\begin{array}{c}
0 \\
-x_{1} W_{1}-\frac{x_{1}}{I_{t 1}}\left(\tau_{e 1}-\tau_{b 2}+\tau_{e 2}\right) \\
0 \\
-x_{2} W_{2}+\frac{x_{2}}{I_{t 1}}\left(\tau_{e 1}-\tau_{b 2}\right)+x_{2} \frac{I_{h 2}+I_{t 1}}{I_{h 2} I_{t 1}} \tau_{e 2}+f_{0} \\
0 \\
W_{1}+\frac{1}{I_{t 1}}\left(\tau_{e 1}-\tau_{b 2}+\tau_{e 2}\right) \\
0 \\
W_{2}-\frac{1}{I_{t 1}}\left(\tau_{e 1}-\tau_{b 2}\right)-\frac{I_{h 2}+I_{t 1}}{I_{h 2} I_{t 1}} \tau_{e 2}
\end{array}\right]
\end{aligned}
$$

where $A_{1}, A_{2}$ are given in equation ( 23$) ; \tau_{e i}, \tau_{b i}$ are defined by equations ( 11$)-(12)$, and $B_{1}, B_{2}, W_{1}, W_{2}, f_{0}$ are given below

$$
\begin{aligned}
& B_{1}=\left[\begin{array}{cccc}
0 & 0 & \frac{x_{1} I_{h 2} \tilde{\beta}}{I_{h 2}+I_{t 1}} & \frac{x_{1} I_{h 2} \tilde{\alpha}}{I_{h 2}+I_{t 1}} \\
0 & 0 & 0 & 0 \\
0 & 0 & -x_{2} \tilde{\beta} & -x_{2} \tilde{\alpha}
\end{array}\right] \\
& B_{2}=\left[\begin{array}{cccc}
0 & 1 & 0 & 0 \\
0 & 0 & \frac{-I_{h 2} \tilde{\beta}}{I_{h 2}+I_{t 1}} & \frac{-I_{h 2} \tilde{\alpha}}{I_{h 2}+I_{t 1}} \\
0 & 0 & 0 & 1 \\
0 & 0 & \tilde{\beta} & \tilde{\alpha}
\end{array}\right] \\
& W_{1}=\left.\frac{I_{h 2}}{\gamma_{2} I_{t 1}} \frac{\left(E I_{2}\left(x_{2}\right) w_{2 x x}\right)_{x x}}{l_{2} \rho_{2}\left(x_{2}\right)}\right|_{x_{2}=l_{2}} \\
& W_{2}=\left.\frac{I_{h 2}+I_{t 1}}{-\gamma_{2} I_{t 1}} \frac{\left(E I_{2}\left(x_{2}\right) w_{2 x x}\right)_{x x}}{l_{2} \rho_{2}\left(x_{2}\right)}\right|_{x_{2}=l_{2}} \\
& f_{0}=\operatorname{Cos}_{2}\left[K_{1} l_{1}\left(\alpha \dot{\theta}_{1}+\beta\left(\theta_{1}-\theta_{1 d}\right)\right)\right. \\
& \left.-\frac{\gamma_{1} l_{1}}{I_{h 1}}\left(\tau_{b 2}-\tau_{e 2}-K \dot{\theta}_{1}\right)\right]+\dot{\theta}_{2} \operatorname{Sin} \theta_{2}\left[\dot{w}_{1}\left(l_{1}, t\right)+l_{1} \dot{\theta}_{1}\right] \\
& \tilde{\beta}=\frac{I_{h 2}+I_{t 1}}{\gamma_{2} I_{t 1}} K_{2} \beta \\
& \tilde{\alpha}=\frac{I_{h 2}+I_{t 1}}{\gamma_{2} I_{t 1}} K_{2} \alpha
\end{aligned}
$$

where $B_{1}, B_{2}$ are finite dimensional linear bounded operators, $f(\mathbf{0})=0$ and $f(\mathbf{z})$ is differentiable. Since $(\lambda I-A)^{-1}$ is compact for $\lambda>\tilde{\alpha}$, (see e.g. [12]), then it follows that the solutions of ( 22) locally exists in; moreover if $\mathbf{z}(0) \in D(A)$, then $\mathbf{z}(t) \in D(A)$ as well. Since the solutions are bounded, see ( 21$)$, it can easily be shown that local solutions can be extended globally as well. In order to complete the proof, we should show that $\dot{V}=0$ implies $\mathbf{z}=0$. If $\dot{V}=0$ then $\delta W_{n c}=0$ since the power associated with nonconservative forces is equal to the time rate of change of the total energy [14]. For the system ( 2 - 10),

$$
\delta W_{n c}=\tau_{1} \delta \theta_{1}+\tau_{2} \delta\left(\theta_{2}-w_{1 x}\left(l_{1}, t\right)\right)
$$

where $\delta \theta_{1}$ and $\delta\left(\theta_{2}-w_{1 x}\left(l_{1}, t\right)\right)$ are arbitrary nonzero variations by definition then $\tau_{1}=0$ and $\tau_{2}=0$. Besides, $\dot{V}=0$ implies $\dot{\theta}_{1}=\ddot{\theta}_{1}=0$ directly and since $\tau_{1}=0$ by ( 4) and ( 11) we obtain $\tau_{b 1}=0$ as well. Using the boundary condition ( 8 ) we get $W_{1}=W_{2}=0$ for $f(\mathbf{z})$. Thus, $W_{1}=W_{2}=0, \quad \ddot{\theta}_{1}=0$ and equations (13), ( 22) implies that $\tau_{b i}=\tau_{e i}=0$. Using these results and the control laws ( 18 - 19) for the boundary condition ( 6) with equation ( 13 ), we have $\tau_{b 2}=-I_{h 2} \ddot{\theta}_{2}$. Equations (5) and (9) give respectively

$$
\tau_{e 2}=-I_{h 2} \ddot{\theta}_{2}=I_{t 2} \ddot{\theta}_{2}
$$

that verifies $\tau_{e 1}=\tau_{b 2}=\tau_{e 2}=\ddot{\theta}_{2}=0$. Thus, the main PDEs ( 2 - 3) become homogeneous such as

$$
\begin{aligned}
& \rho_{1}\left(x_{1}\right) \ddot{w}_{1}+\left(E I_{1}\left(x_{1}\right) w_{1 x x}\right)_{x x}=0 \\
& \rho_{2}\left(x_{2}\right) \ddot{w}_{2}+\left(E I_{2}\left(x_{2}\right) w_{2 x x}\right)_{x x}=0
\end{aligned}
$$

where flexural deflection $w_{i}(x, t)$ can be expressed by separation of variables such that [18]

$$
w_{i}(x, t)=\phi_{i}(x) e^{\lambda_{i} t}
$$

where $i=1,2 ; \lambda_{i}$ 's are nonzero complex eigenvalues and $\phi_{i}$ 's are eigenfunctions for equations ( 26 - 27) with the above boundary conditions that are derived for $\dot{V}=0$. Thus, equations ( 26 - 27) become variable coefficient, ordinary differential equations of order four, such as [18]

$$
\begin{aligned}
& \lambda_{1}^{2} \rho_{1}\left(x_{1}\right) \phi_{1}\left(x_{1}\right)+\frac{d}{d x_{1}^{2}}\left(E I_{1}\left(x_{1}\right) \frac{d}{d x_{1}^{2}} \phi_{1}\left(x_{1}\right)\right)=0 \\
& \lambda_{2}^{2} \rho_{2}\left(x_{2}\right) \phi_{2}\left(x_{2}\right)+\frac{d}{d x_{2}^{2}}\left(E I_{2}\left(x_{2}\right) \frac{d}{d x_{2}^{2}} \phi_{2}\left(x_{2}\right)\right)=0
\end{aligned}
$$

For nonuniform $i^{\text {th }}$ link, $\phi_{i}(x)$ can be defined as

$$
\begin{aligned}
\phi_{i}(x) & =p_{i}(x) r_{i}(x) \\
\phi_{i x}(x) & =p_{i x}(x) r_{i}(x)+p_{i}(x) r_{i x}(x) \\
\phi_{i x x}(x) & =p_{i x x}(x) r_{i}(x)+2 p_{i x}(x) r_{i x}(x) \\
& +p_{i}(x) r_{i x x}(x) \\
\phi_{i x x x}(x) & =p_{i x x x}(x) r_{i}(x)+3 p_{i x x}(x) r_{i x}(x) \\
& +3 p_{i x}(x) r_{i x x}(x)+p_{i}(x) r_{i x x x}(x) \\
\phi_{i x x x x}(x) & =p_{i x x x x}(x) r_{i}(x)+4 p_{i x x x}(x) r_{i x}(x) \\
& +6 p_{i x x}(x) r_{i x x}(x)+4 p_{i x}(x) r_{i x x x}(x) \\
& +p_{i}(x) r_{i x x x x}(x)
\end{aligned}
$$

where $p_{i}(x)=a_{i} x^{4}+b_{i} x^{3}+c_{i} x^{2}+d_{i} x+e_{i}$ and $r_{i}(x)$ is possibly nonlinear function which is the fourth order differentiable at least and satisfies the conditions such that $r_{i}(0) \neq 0, \quad r_{i}(l i) \neq 0$. Then the coefficients of 
the polynomial $p_{i}(x)$ are obtained by using the boundary conditions and the dominant control laws such as

$$
\begin{aligned}
\phi_{i}(0) & =0 \rightarrow e_{i}=0 \text { from } \quad(10) \\
\phi_{i x}(0) & =0 \rightarrow d_{i}=0 \text { from }(10) \\
\phi_{i}\left(l_{i}\right) & =0 \\
\phi_{i x}\left(l_{i}\right) & =0 \quad \text { see } \text { Remark } 3.2 \\
\phi_{i x x}(0) & =0 \rightarrow c_{i}=0 \quad \text { from } \tau_{b i}=0 \\
\phi_{i x x}\left(l_{i}\right) & =0 \rightarrow 12 a_{i} l_{i}^{2}+6 b_{i} l_{i}=0 \text { from } \tau_{e i}=0 \\
\phi_{i x x x}\left(l_{i}\right) & =0 \rightarrow 24 a_{i} l_{i}+6 b_{i}=0 \\
\phi_{i x x x x}\left(l_{i}\right) & =0 \rightarrow 24 a_{i}=0
\end{aligned}
$$

thus $a_{i}=b_{i}=0$ while $\rho_{i}\left(x_{i}\right), E I_{i}\left(x_{i}\right), E I_{i x}\left(x_{i}\right)$ and $E I_{i x x}\left(x_{i}\right)$ are assumed to be nonzero at $x_{i}=0$, and $x_{i}=$ $l_{i}$. Consequently, $p_{i}(x)=0, \phi_{i}(x)=0$, then $w_{i}=\dot{w}_{i}=0$ for $\dot{V}=0$. On the other hand, using the dominant control laws ( 14 - 15) and the above results that include $\left(\tau_{b i}=\right.$ $0, \tau_{e i}=0$ ), and equations (4), (5) yields $\ddot{w}_{i}\left(l_{i}, t\right)=0$, we also have $\dot{\theta}_{1}=\dot{\theta}_{2}=0$ and $\theta_{1}=\theta_{1 d}, \quad \theta_{2}=\theta_{2 d}$. Consequently, $\dot{V}=0$ really implies $\mathbf{z}=0$.

In the light of [10, Theorem 3.64 and 3.65], the closedloop system ( 2 - 19) is asymptotically stable. $\diamond$

Remark 3.2: Since we have already neglected $w_{1 x}$, we have also omitted $\ddot{w}_{1 x}$ in the final calculations. Note that such terms are also omitted in [13]. Note that although in [13] two damping terms are introduced in the simulations, we do not use any damping term in our case.

\section{Simulation Results}

\begin{tabular}{|l|l|}
\hline Parameter & Value \\
\hline Length of links, & $l_{1}=0.5 \mathrm{~m}, l_{2}=0.6 \mathrm{~m}$ \\
\hline Time step & $\Delta t=3 e-5 \mathrm{sec}$ \\
\hline Spatial steps & $\Delta x_{1}=l_{1} / 20$, \\
\hline & $\Delta x_{2}=l_{2} / 20$ \\
\hline Young's Modulus, $E$ & $70 \quad \mathrm{GPa}$ \\
\hline Density, $\rho$ & $2742 \quad \mathrm{kgm}^{-3}$ \\
\hline Thickness of links $(\mathrm{m})$ & $c_{1}=0.003175$, \\
\hline & $c_{2}=0.00238$ \\
\hline $\begin{array}{l}\text { Maximum height for tapering } \\
\text { at the root of the link }\end{array}$ & $b_{o}=0.0654 \mathrm{~m}$ \\
\hline Linear slope for tapering & $a_{1}=0.04$ \\
\hline Hub inertias $\left(\mathrm{kgm}^{2}\right)$ & $I_{h 1}=0.0055$ \\
\hline & $I_{h 2}=0.0068$ \\
\hline Tip inertias, $\left(\mathrm{kgm} \mathrm{m}^{2}\right)$ & $I_{t 1}=0.0139$, \\
\hline & $I_{t 2}=0.00024$ \\
\hline Hub mass $(\mathrm{kg})$ & $m_{h 2}=0.678$ \\
\hline Tip mass, $(\mathrm{kg})$ & $m_{t 1}=0.981$, \\
\hline & $m_{t 2}=0.204$ \\
\hline$\theta_{1 d}$ (desired) & $\pi / 2 \quad \mathrm{rad}$ \\
\hline$\theta_{2 d}$ (desired) & $-\pi / 2 \quad \mathrm{rad}$ \\
\hline$K_{1}=.007, \quad K_{2}=0.01$ & $\alpha=600, \quad \beta=800$ \\
\hline$\gamma_{1}=1 e-3, \quad \gamma_{2}=1 e-4$ & $K=9$ \\
\hline
\end{tabular}

TABLE II

Parameters of the Flexible Arm.

The proposed control scheme is tested with the simulation program implemented in MATLAB. The PDEs are discretized in the space domain by the finite difference

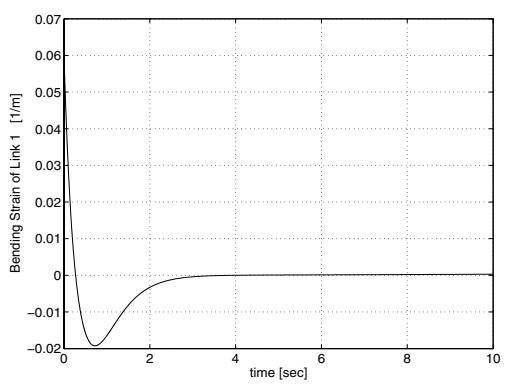

Fig. 2. Bending strain at the end of the link 1

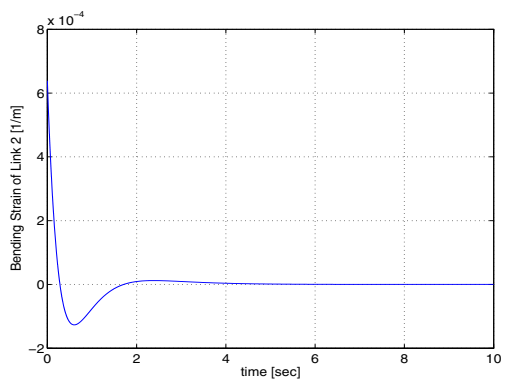

Fig. 3. Bending strain at the end of the link 2

method, to obtain ODEs at each of the nodes. Then, ODEs are solved numerically. Instead of dealing with complexity of the fourth order derivative approximation, the second order derivative approximation has been used with the help of auxiliary states. Those states are more meaningful in a real problem as well since they corresponds to physical variables such as deflections, velocity and bending moments [20]. However, the number of ODEs to solve and the computation time are increased in return of the robust stability of the numeric scheme. The parameters used in the model for system ( 2 - 10), partially given in [13] are listed in TABLE II.

Although the control setup in the previous section is given for links that can have any kind of variable cross-section; in this particular simulation, rectangular cross-sections of given uniform thickness are used. For small values of tip mass and tip inertia moment relative to the ones for beam, the optimum shape is approximately a linearly tapered beam [8]. Therefore, the height $b_{i}(x)$, density $\rho_{i}(x)$ and cross-section area moment $I_{i}(x)$ at any point can be calculated with the parameters given in TABLE II such as

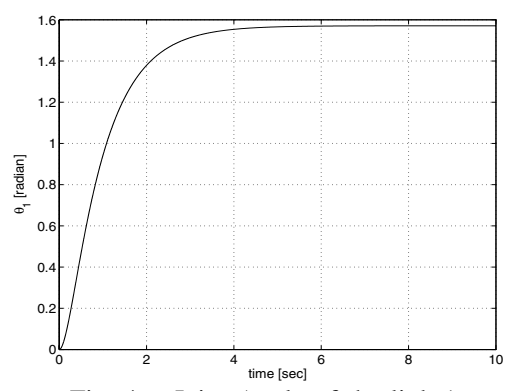

Fig. 4. Joint Angle of the link 1 


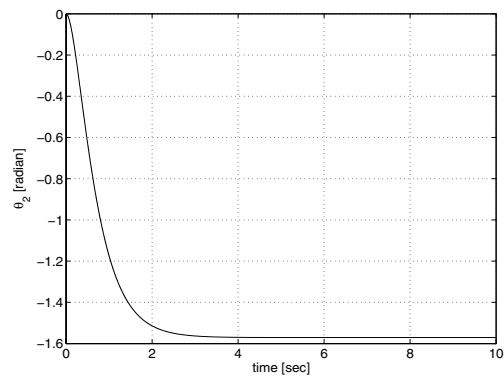

Fig. 5. Joint Angle of the link 2

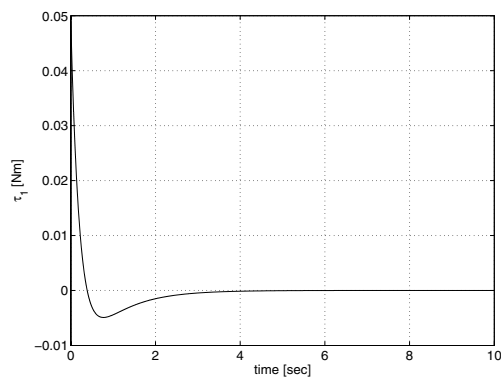

Fig. 6. Control Torque of Joint 1

$$
\begin{aligned}
b_{i}(x) & =b_{o}-2 l_{i} a_{1}+2\left(l_{i}-x\right) a_{1} \\
\rho_{i}(x) & =\rho b_{i}(x) c_{i} \\
I_{i}(x) & =b_{i}(x) c_{i}^{3} / 12 .
\end{aligned}
$$

The simulation results are presented in Figures 2 - 7. Smooth time histories of all variables of interest without overshoot show the effectiveness of the controller performance especially with such demanding desired positions $\left(\theta_{1 d}=\pi / 2\right.$ and $\left.\theta_{2 d}=-\pi / 2\right)$. Comparing the time responses for FEM (Finite Element Method) case in [21] with the simulation results for PDE, it is observed that the required control energy in PDE cases is much less than the one in FEM due to the exactness of PDE. Also, PDE responses are smoother than the ones in FEM approach, and have no overshoot or no chattering for all states.

\section{Conclusions}

It has been shown by the simulation results that the new controller design method can provide asymptotic stability of flexural modes and set-point regulation of rigid

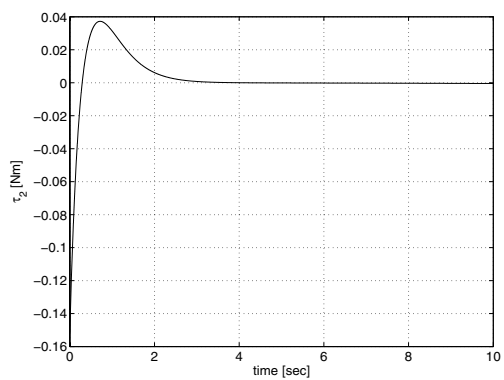

Fig. 7. Control Torque of Joint 2 modes simultaneously. In the proof of the main theorem, infinite dimensionality of the problem has been retained as opposed to other energy-based approaches for multi-link robot arms in the literature. In future, compensation of variable tip mass would be an extension to the proposed controller that already manages the nonuniform flexible robot arms successfully.

\section{REFERENCES}

[1] M.W. Vandegrift, F.L. Lewis, and S.Q. Zhu, 'Flexible-Link robot arm control by a feedback linearization/Singular perturbation approach', Journal of Robotic Systems, vol. 11(7), pp. 591-603, 1994.

[2] M.R. Rokui and K. Khorasani,'Experimental results on discretetime nonlinear adaptive tracking control of a Flexible-link Manipulator', IEEE Transactions on systems, man, and cybernetics, vol.30/1, pp.151-164, 2000.

[3] B. Siciliano and L. Villani, 'Two-Time scale force and position control of flexible manipulators',Proceedings of the IEEE International Conference on Robotics and Automation, pp. 2729-2734,Seoul, 2001.

[4] X. Zhang,W. Xu,S.S. Nair,'PDE modeling and control of a flexible two-link manipulator', Proceedings of the American Control Conference, pp. 3796-3801,Anchorage, 2002.

[5] F.J. Raab, D.J. Trudnowski,'Experiments in two-axis vibration damping using inertial torques through momentum wheel control',Proceedings of the American Control Conference, pp. 34773481,Philadelphia, 1998.

[6] Ye Zhu, Jinhao Qiu, Junji Tani,'Simultaneous Optimization of a Two-link Flexible Robot Arm',Journal of Robotic Systems, vol. 18(1), pp. 29-38, 2001.

[7] M. Moallem, R.V. Patel, K. Khorasani, 'Optimization of an Actuated Flexible Arm for Improved Control Properties', Proceedings of the IEEE International Conference on Control Applications, pp. 709-714,Anchorage, 2000.

[8] F.Y. Wang and J.L. Russell, 'Optimum Shape Construction of Flexible Manipulators with Tip Loads',Proceedings of the 31st IEEE Conference on Decision and Control, pp.311-316, Tucson, 1992.

[9] F.Y. Wang and Y. Gao , Advanced Studies of Flexible Robotic Manipulators, New Jersey: World Scientific, 2003.

[10] Z. H. Luo, B. Z. Guo and Ö. Morgül, Stability and Stabilization of Infinite Dimensional Systems with Applications, London: SpringerVerlag, 1999

[11] Ö. Morgül, 'Orientation and Stabilization of a Flexible Beam Attached to a Rigid Body: Planar Motion', IEEE Transactions on Automatic Control, vol.36, No.8, pp.953-962, 1991.

[12] B. Z. Guo, 'Riesz Basis Property and Exponential Stability of Controlled Euler-Bernoulli Beam Equations with Variable Coefficients', SIAM Journal of Control Optim., vol.40, No.6, pp.1905-1923, 2002.

[13] X. Zhang, 'Multi-scale System Dynamics and Control-Two Case Studies', May 2001, Ph.D. dissertation, University of MissouriColumbia.

[14] L. Meirovitch, Fundamentals of Vibrations, New York: McGrawHill, 2001.

[15] J.N. Reddy, An Introduction to the Finite Element Method, New York: McGraw-Hill, 1993.

[16] G.G. Hastings and W.J.Book, 'A Linear Dynamic Model for Flexible Robotic Manipulators',IEEE Control System Magazine, vol.7, pp.61-64, 1987.

[17] J.P. Den Hartog, Mechanical Vibrations, New York: Dover, 1985.

[18] H. Baruh, Analytical Dynamics, Boston: McGraw-Hill, 1999.

[19] T.Y. Yang, Finite Element Structural Analysis, New Jersey: Prentice Hall, 1986.

[20] N.S. Abhyankar, E.K. Hall II, S.V. Hanagud, 'Chaotic Vibrations of Beams:Numerical Solution of Partial Differential Equations',ASME Journal of Applied Mechanics, vol.60, pp.167-174, 1993.

[21] Dog̃an, M. and Y. Istefanopulos, 'Optimal Nonlinear Control of Two-Link Flexible Arm with Adaptive Internal Model, Proc. 44th IEEE Conference On Decision and Control, and the European Control Conference, pp. 771-776, Seville, Spain, 2005. 